\title{
REVISIÓN DE LAS INVESTIGACIONES A NIVEL MOLECULAR SOBRE EL IMPACTO DEL FUEGO EN LA MATERIA ORGÁNICA DEL SUELO: PROGRESOS EN EL SIGLO XXI
}

\author{
J.M. DE LA ROSA ${ }^{1}$, H. KNICKER ${ }^{1}$, J.A. GONZÁLEZ-PÉREZ ${ }^{1}$, \\ F.J. GONZÁLEZ-VILA ${ }^{1}$, N.T. JIMÉNEZ-MORILLO ${ }^{1}$, G. ALMENDROS ${ }^{2}$ \\ ${ }^{1}$ Instituto de Recursos Naturales y Agrobiología de Sevilla (IRNAS-CSIC). \\ Avenida de la Reina Mercedes 10, 41012 Sevilla. \\ ${ }^{2}$ Museo Nacional de Ciencias Naturales (MNCN-CSIC). Serrano 115b, 28006 Madrid.
}

\begin{abstract}
RESUMEN. Los incendios forestales son un factor de perturbación importante en muchos ecosistemas, especialmente en las áreas Mediterráneas. Es bien conocido que los incendios forestales producen cambios significativos en la materia orgánica del suelo (MOS), tanto a nivel cualitativo como cuantitativo. Estos cambios, a su vez, afectan propiedades relevantes y estrechamente relacionadas con la MOS, como es el caso de su respuesta frente a la erosión, su capacidad de retención hídrica o la agregación del suelo. Los efectos de las elevadas temperaturas en la MOS son altamente variables y dependen de varios factores interrelacionados. Por todo ello, el contenido en MOS puede variar desde una desaparición casi total a un notable incremento debido a la incorporación de necromasa procedente de la vegetación parcialmente carbonizada. Además de la pérdida de C orgánico o del incremento debido a la necromasa acumulada en el suelo, la MOS preexistente en el suelo transformarse en formas pirogénicas de $C$ debido al efecto de las altas temperaturas; estas formas de material pirogénico comúnmente denominadas "black carbon" o carbono negro, han sido descritas desde los años 90 como formas de materia orgánica (MO) altamente condensadas, considerándose que presentan un carácter recalcitrante frente a la degradación biológica, por lo que podrían contribuir significativamente al secuestro efectivo de $C$ y $N$ a largo plazo en el suelo. Este trabajo presenta una revisión práctica de los conocimientos aportados durante los últimos diez años en la investigación dedicada al estudio del impacto del fuego sobre la estructura molecular de la MOS. Durante este periodo, algunas metodologías se han optimizado y son hoy en día de utilidad en la caracterización de cambios producidos por el fuego a nivel molecular en la MOS. Por otra parte, han aparecido herramientas analíticas para predecir los índices de recuperación de la calidad de la MOS en los suelos afectados por incendios. Finalmente, algunos estudios recientes parecen indicar que la MOS pirogénica generada por efecto del fuego es, en muchos casos, considerablemente menos recalcitrante de lo que se pensaba.
\end{abstract}


Revisiting molecular-level research on the impact of fire on soil organic matter: Progresses in the 21st century

ABSTRACT. Wildfires represent an important disturbance in many ecosystems, especially in Mediterranean areas. It is well known that forest fires exert dramatic changes in soil organic matter (SOM) quality and quantity that in turn affect relevant and closely related characteristics like soil runoff and erosion response, through changes on factors like soil wettability and aggregation. The effects of severe heating in SOM are highly variable and depend on many interrelated factors. Therefore, SOM content may be impacted from its almost total removal to significant increases as a consequence of external inputs, mainly from forest necromass. Additionally to $C$ losses or exogenous increases, pre-existing $C$ forms in the soil may be transformed into recalcitrant pyrogenic material such as black carbon presumably contributing to the long-term $C$ and $N$ sequestration in soils. This paper presents a practical revision of the progresses during the last decade on the studies of the fire impacts on the SOM. During this period, several analytical methodologies have been improved to characterise molecular changes into the SOM due to fire. Other tools have appeared to predict soil recovery indexes after a wildfire event. On the other hand, recent studies have reported that pyrogenic SOM resulting from fires may be less recalcitrant than previously assumed.

Palabras clave: materia orgánica del suelo, fuego, carbón pirogénico, intensidad del fuego, secuestro de $\mathrm{C}$ en el suelo.

Key words: soil organic matter, wildfire, pyrogenic carbon, fire intensity, soil carbon sequestration.

Recibido el 3 de diciembre de 2013

Aceptado el 4 de febrero de 2014

*Correspondencia: Instituto de Recursos Naturales y Agrobiología de Sevilla (IRNASCSIC). Avenida de la Reina Mercedes 10, 41012 Sevilla. E-mail: jmrosa@irnase.csic.es

\section{Introducción}

Los incendios forestales han estado históricamente presentes en mayor o menor medida en todos los bosques Mediterráneos (Pausas et al., 2008; LasherasÁlvarez et al., 2013). Sin embargo el incremento tanto en el número de incendios como en el área quemada, debido posiblemente al aumento en la temperatura media global durante el Siglo XX y a los frecuentes períodos de sequía (Moriondo et al., 2006), han convertido al fuego en una de las principales amenazas de estos ecosistemas. La consecuencia más obvia de un incendio forestal es la pérdida parcial o completa de la vegetación, que determina diversas consecuencias económicas y medioambientales. Sin embargo, el fuego es, con mucha frecuencia, responsable de severos cambios en las propiedades físicas y químicas de los suelos. En el caso de 
la materia orgánica del suelo (MOS) se han observado cambios en los contenidos totales de carbono $(\mathrm{C})$ y nitrógeno $(\mathrm{N})$, alteraciones estructurales en las fracciones húmicas, descenso en su biodegrabilidad, incremento en la hidrofobicidad, etc. (González-Pérez et al., 2004; Almendros y González-Vila, 2012; Martín et al., 2012). Dichos cambios presentan gran importancia a nivel global, puesto que la MOS constituye la mayor reserva de $\mathrm{C}$ de la superficie de la Tierra, de tal forma que incluso pequeñas alteraciones en su contenido total y sobre todo en su biodegradabilidad, se traducen en cambios notables en los balances de materia y energía de los ciclos del C. En un incendio forestal se liberan a la atmósfera grandes cantidades de $\mathrm{CO}_{2}$. El modelo más sencillo de combustión de la biomasa asume que los productos finales son $\mathrm{H}_{2} \mathrm{O}, \mathrm{CO}_{2}$ y los minerales contenidos en las cenizas. Sin embargo, bajo condiciones naturales, la combustión de la materia orgánica (MO) es frecuentemente incompleta, sobre todo en los primeros centímetros de profundidad, por lo que además de gran cantidad de $\mathrm{CO}$ y $\mathrm{CH}_{4}$, se desprende una mezcla compleja de productos de pirólisis que incluyen hidrocarburos policíclicos aromáticos y otros productos orgánicos muy diversos. Muchos de estos compuestos han sido identificados como recalcitrantes frente a la degradación biológica.

Los modelos para explicar la dinámica de la MOS clasifican la fracción orgánica según tres velocidades de descomposición: activa, lenta y pasiva (GonzálezVila y Almendros, 2013). En el compartimento activo, el ciclo de retorno se mide en años y está formada por la MO biológicamente activa y fácilmente biodegradable. La fracción lenta corresponde a la MO con un ciclo de retorno intermedio (décadas) y el compartimento pasivo lo constituye la MO más recalcitrante, con una tasa de retorno de cientos a miles de años (Wander, 2004). Esta última fracción más recalcitrante, asociada a los comportamientos lento y pasivo, es más importante en procesos fisicoquímicos, tales como la adsorción, la capacidad de intercambio catiónico y retención de agua, así como en el secuestro de C orgánico a largo plazo (Wander, 2004). Las macromoléculas más resistentes contienen anillos aromáticos, como en el caso de la lignina, y estructuras polimetilénicas, como algunos polímeros lipídicos y las ceras (Lützow et al., 2006). Por todo ello, los incendios forestales se encuentran estrechamente relacionados con los mecanismos de secuestro de $\mathrm{C}$ y de $\mathrm{N}$ en los suelos forestales (Parker et al., 2001). También se evidencian cambios significativos a nivel molecular en la MO de los suelos afectados por incendios: además del aporte de material carbonizado y potencialmente refractario procedente de la biomasa vegetal, se ha descrito una destrucción selectiva de las estructuras menos resistentes a la degradación térmica, y se ha demostrado la neoformación de estructuras aromáticas mediante reacciones de condensación que incluyen la formación de compuestos heterocíclicos de $\mathrm{N}$ (Almendros et al., 2003; González-Pérez et al., 2004; Knicker, 2007; De la Rosa et al., 2008a) no existentes en la biomasa antes del efecto del fuego. Esta última circunstancia merece ser resaltada, pues con independencia de la destrucción de MO en forma de $\mathrm{CO}_{2}$ y agua, y desde el punto de vista estructural, se produce un incremento considerable en la complejidad molecular de la MO en vez de una simplificación, toda vez que se generan abióticamente compuestos no pre-existentes 
en el suelo, e incluso se condensan compuestos de bajo peso molecular para formar macromoléculas (González-Vila y Almendros, 2013), en contraposición a la idea de que el fuego podría tener un efecto predominante de fragmentación de bio- y geomacromoléculas. Estas modificaciones se traducen en efectos específicos en la calidad de la MO (fundamentalmente se incrementa su resistencia a la biodegradación y disminuye su solubilidad tanto en solventes polares como apolares) lo que se manifiesta en propiedades de fácil determinación como son el frecuente incremento en la hidrofobicidad, la disminución o desaparición de sus propiedades coloidales y los cambios en su resistencia a la alteración biológica o química. Estos cambios van frecuentemente asociados a la desorganización de la estructura del suelo, y en muchas ocasiones (sobre todo en suelos con niveles elevados de MO) al aumento en la repelencia al agua (Bodí et al., 2012), haciéndolo más susceptible a la erosión (Doerr et al., 2009).

El uso combinado de técnicas analíticas espectroscópicas y cromatográficas se ha mostrado de gran utilidad para la evaluar los cambios en la composición y estructura de la MOS debidos al fuego (González-Pérez et al., 2004; Knicker et al., 2005; Tinoco et al., 2006; De la Rosa et al., 2008a; 2013), sin embargo requiere en muchos casos de instrumentación científica de elevado coste así como de considerable tiempo de preparación en laboratorio, por lo que no son de aplicación habitual. Este trabajo presenta varias técnicas analíticas destinadas a reconocer los cambios ocurridos en la MOS a nivel molecular, en particular los que han experimentado un notable desarrollo durante la última década.

\section{La materia orgánica del suelo y el fuego}

La MOS ha sido considerada un componente esencial de los suelos desde los orígenes de la Edafología como ciencia independiente. Tanto la génesis como la evolución de los distintos tipos de suelo dependen fundamentalmente de la acumulación de MO, de la formación de un complejo de alteración que incluye la modificación de los componentes minerales (horizonte B), y de una translocación de materiales a lo largo del perfil. Desde el punto de vista cualitativo, la MOS influye en sus propiedades físicas y químicas, así como en la disponibilidad de nutrientes para las plantas y el desarrollo de la biomasa microbiana. Debido a la amplia distribución espacial y su elevada funcionalidad (de tipo biológico, físico y químico), la MOS participa en mayor o menor medida en la totalidad de los procesos biogeoquímicos, que a su vez inciden en la productividad y la conservación de los ecosistemas terrestres, con especial referencia a la estabilidad estructural del suelo y el control de la erosión (Stevenson, 1994). Por todo ello, la MO constituye un factor determinante de la calidad y la "salud" de los suelos, entendida como el conjunto de "características biológicas, físicas y químicas que son esenciales para una productividad sostenible con el mínimo impacto ambiental desfavorable" (Arias et al., 2005). Por otra parte, la MOS determina la calidad de los ecosistemas, al influir en la composición de la solución del suelo, que a su vez se encuentra 
estrechamente relacionada tanto con la fertilidad de los suelos como con la calidad de las aguas superficiales y subterráneas.

Los incendios forestales, así como las prácticas agronómicas basadas en la aplicación del fuego, tales como las quemas de matorral o de residuos de cosechas, que se han aplicado durante milenios en muchas regiones agrícolas de la Península Ibérica, afectan simultáneamente a la calidad y cantidad de MOS, y pueden determinar cambios lentamente reversibles en el balance de $\mathrm{C}$ del suelo. El estudio de los efectos de los incendios sobre la MOS resulta muy complejo. Por una parte, la intensidad y duración de las alteraciones dependerá directamente de la severidad del incendio, controlada a su vez por factores ambientales tales como la cantidad, naturaleza y humedad de la vegetación (combustible natural), la temperatura y grado de humedad del aire y el suelo, la velocidad del viento o la topografía (Certini, 2005).

\section{Contenido en carbono y nitrógeno orgánico}

La influencia del fuego sobre el contenido en $\mathrm{C}$ y $\mathrm{N}$ orgánicos del suelo ha sido ampliamente estudiada (González-Pérez et al., 2004; Kavdir et al., 2005; Knicker et al., 2005; Tinoco et al., 2006). Los resultados son muy variables y se encuentran directamente vinculados a la intensidad del fuego. El cambio más esperable que podría suponerse en el suelo debido a un incendio sería la pérdida de MO. Sin embargo, esta alteración dependerá en gran medida de la severidad del fuego. Sólo en incendios de alta intensidad se ha descrito la pérdida de la práctica totalidad de la MO en el horizonte superficial del suelo. Sin embargo, en incendios de media y baja intensidad, el contenido en $\mathrm{C}$ puede aumentar significativamente debido a la incorporación masiva al suelo de necromasa, que incluye tanto la biomasa subterránea de vegetales muertos, como material vegetal parcialmente carbonizado proveniente de las partes aéreas de la vegetación quemada (Fig. 1). En términos generales, y dependiendo del tiempo de calentamiento, y de la destilación de compuestos volátiles, se estima que la pérdida de $\mathrm{C}$ orgánico comienza a temperaturas entre 100 y $200^{\circ} \mathrm{C}$, después de eliminarse el agua higroscópica y constitucional. Algunos trabajos recientes han señalado que a medio o largo plazo los incendios tienden a incrementar el contenido total de $\mathrm{C}$ del suelo en el horizonte superficial (Johnson y Curtis, 2001; Certini et al., 2005). Las razones sugeridas para explicar este incremento han sido: i) la incorporación a la fracción mineral del suelo de residuos no quemados, que consecuentemente están más protegidos de la descomposición bioquímica, ii) la colonización de la zona quemada por vegetación de rápido crecimiento, unido al desarrollo de especies fijadoras de $\mathrm{N}$, que se ha demostrado que pueden ayudar al secuestro de C, y iii) la transformación de la MO fresca a formas más recalcitrantes (este cambio será abordado en el siguiente apartado de este artículo). En cuanto a la relación $\mathrm{C} / \mathrm{N}$, suele disminuir en las muestras afectadas por incendios (González-Pérez et al., 2004; De la Rosa et al., 2009), probablemente debido a la formación "de novo" de formas heterocíclicas nitrogenadas recalcitrantes (Almendros et al., 2003; Knicker et al., 2005). 


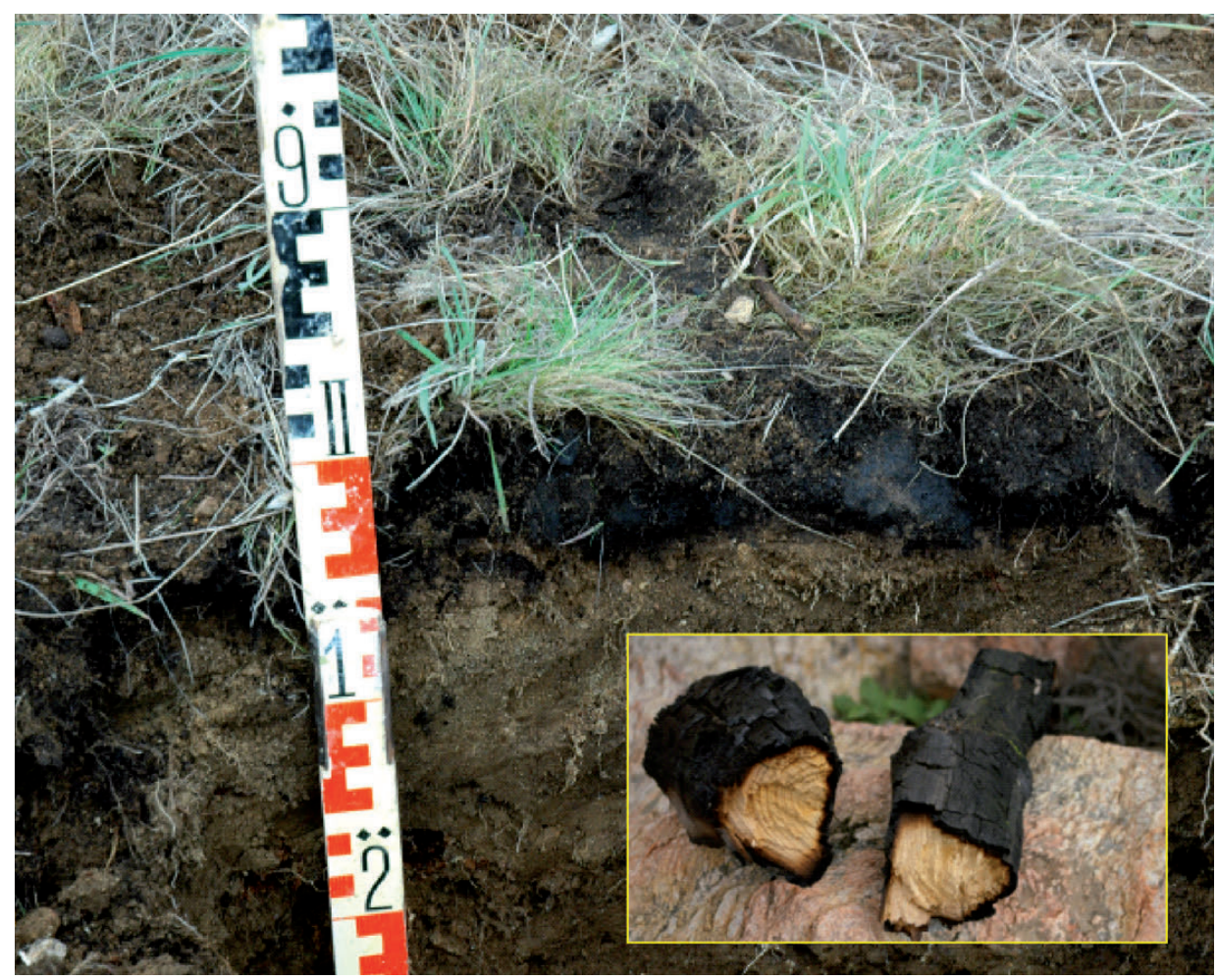

Figura 1. Perfil de suelo afectado por incendio en la Sierra de Madrid (2005) y biomasa local. Fotos: H. Knicker y G. Almendros.

En cuanto a la estabilización de $\mathrm{C}$ debido al fuego, los efectos pueden ser muy diferentes en función del tipo de ecosistema (Kuhlbusch y Crutzen, 1995). Los ecosistemas de alta actividad biológica (ej. bosques tropicales) pueden, en la práctica, tener un balance cero o incluso negativo en lo que concierne al secuestro de $\mathrm{C}$ debido a la actividad y rapidez de las transferencias de $\mathrm{C}$ y a que el $\mathrm{C}$ atmosférico se encuentra más "secuestrado" en la biomasa que en los suelos de estos ecosistemas. Sin embargo, en ecosistemas semiáridos (ej. bosque Mediterráneo) la acumulación de las formas estables de C (black carbon) y MO recalcitrante procedentes de incendios en el suelo no es fácilmente accesible a la degradación enzimática, en este caso los procesos abióticos son responsables de la mayor parte de la MO estabilizada en el suelo a lo que contribuye las interacciones órgano minerales estables que son favorecidas en los procesos cíclicos de secado-mojado frecuentes en estos ecosistemas.

\section{Cambios en la composición molecular de la materia orgánica}

Desde los primeros estudios sobre el efecto del fuego en la MOS se ha prestado especial atención a las alteraciones que produce en la composición molecular de la MO, considerando que posiblemente se trata de cambios en la calidad y no en la cantidad total de MO las que podrían explicar las propiedades y el funcionamiento de los suelos tras la inciden- 
cia del fuego. Sin embargo, la participación simultánea de diferentes procesos durante un incendio forestal hace que sea extremadamente difícil cuantificar e incluso reconocer los mecanismos que participan en la transformación de la MOS. Normalmente tiene lugar de forma simultánea la degradación y generación de la MO, de tal forma que el balance entre uno y otro proceso dependerá tanto de la temperatura como del tiempo de calentamiento, así como de la naturaleza de la MO preexistente en el suelo y su grado de asociación con la fracción mineral.

No existe hoy en día un único método de análisis que permita determinar el continuo de materiales formados por el efecto del fuego. Por ello, se hace imprescindible el uso combinado de varias metodologías analíticas.

Los métodos convencionales de análisis físico y químico permiten evaluar la incidencia del calentamiento en las propiedades generales de la MO. Sin embargo, para poder estudiar los cambios estructurales a nivel molecular es necesario combinar técnicas destructivas (tales como los procedimientos de degradación térmica y química) con métodos de caracterización no destructivos (principalmente espectroscópicos).

Las alteraciones que el fuego puede causar en las estructuras moleculares que forman parte de la MOS dependerán de la temperatura y el tiempo a que esta MO sea sometida, es decir, de la intensidad del fuego. Entre 130 y $190^{\circ} \mathrm{C}$ las ligninas y las hemicelulosas empiezan a ser degradadas (Chandler et al., 1983) aunque hay estructuras lignocelulósicas que resisten temperaturas superiores a $450^{\circ} \mathrm{C}$. Por encima de $200^{\circ} \mathrm{C}$ empiezan los procesos de "charring" (De la Rosa et al., 2008b), se trata de procesos carbonización que eliminan el hidrógeno y el oxígeno. Según demostraron Almendros et al. (1992), cuando se alcanzan temperaturas por encima de $\operatorname{los} 300^{\circ} \mathrm{C}$ se producen cambios estructurales de las sustancias húmicas del suelo, previamente descarboxiladas a bajas temperaturas, se producen ciclaciones de forma que los residuos están compuestos principalmente por estructuras carbonosas aromáticas (fundamentalmente "black carbon") altamente resistentes a la oxidación (Knicker et al., 2005) y no existentes en la muestra calentada a temperaturas más bajas. Por ello, el cambio más habitual es el incremento en el contenido de formas de "black carbon" (Rumpel et al., 2007; De la Rosa et al., 2008b; Rovira et al., 2012).

Estos cambios provocan la aparición de nuevos tipos de sustancias húmicas y, en general, del llamado humus piromórfico, término utilizado por Almendros et al. (1984) para hacer referencia a un material humificado formado por MO altamente transformada por efecto de la temperatura, generalmente de propiedades coloidales atenuadas y con alta resistencia a la degradación. Posiblemente la característica estructural de mayor valor diagnóstico para reconocer el impacto del fuego sobre la MOS sea la acumulación de formas heterocíclicas de $\mathrm{N}$ no existentes en los suelos no afectados por el fuego. En particular, la aplicación de ${ }^{13} \mathrm{C}$ y ${ }^{15} \mathrm{~N}$ RMN en estado sólido, ha sido fundamental a la hora de confirmar no solo el incremento cuantitativo sino también la neoformación de estructuras aromáticas y de formas heterocíclicas de N, fundamentalmente pirrólicas, en suelos afectados por incendios y que no se encuentran en proporciones detectables por ${ }^{15} \mathrm{~N}$ NMR en suelos no afectados por quemas, confirmando su origen pirogénico (Fig. 2). Por otra parte, las elevadas temperaturas determinan reacciones de deshidratación y ciclación, con generación de compuestos reactivos y radicales libres que dan lugar a estructuras de $\mathrm{C}$ y $\mathrm{N}$ altamente condensadas, generalmente recalcitrantes (Knicker, 2000). 
En cuanto a la estabilidad a largo plazo de estas formas condensadas piromórficas, estudios recientes de incubación en invernadero de formas de MO pirogénica han cuestionado esta suposición (De la Rosa y Knicker, 2011; Hilscher y Knicker, 2011).
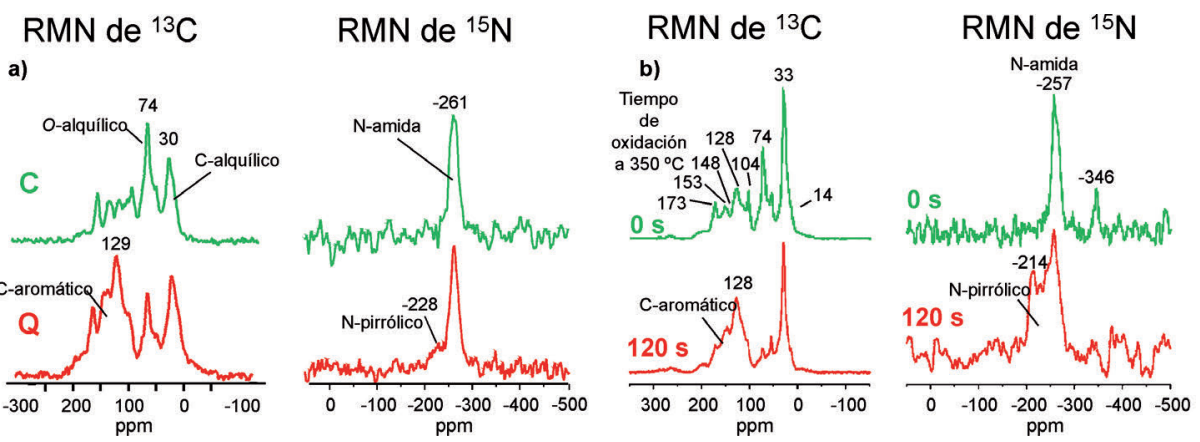

Figura 2. Espectros de Resonancia Magnética Nuclear de ${ }^{13} \mathrm{C} y{ }^{15} \mathrm{~N}$ de: a) Suelos afectados (Q) y no afectados $(C)$ por incendio procedentes de la Sierra de Nerva (Huelva, España). b) Muestras de turba sometidas a calentamiento bajo condiciones controladas $\left(350^{\circ} \mathrm{C} ; 0-120 \mathrm{~s}\right)$. Tanto $Q$ como $C$ presentaban similares condiciones fisiográficas y ambientales.

Las técnicas de análisis térmico (termogravimetría, calorimetría, etc), que han sido utilizadas durante décadas para determinar propiedades básicas del suelo, se han mostrado muy eficaces para medir los cambios en la estabilidad térmica de la MOS debido al efecto de las altas temperaturas (Plante et al., 2009; Alexis et al., 2010; Barros et al., 2011; Fernández et al., 2011), además consiguen distinguir la MO pirogénica y las señales correspondientes a "black carbon" (Fig. 3) del resto de fracciones de MO (Leifeld, 2007; De la Rosa et al., 2008b; Reeves III et al., 2008) y no es necesario pretratamiento alguno de las muestras.

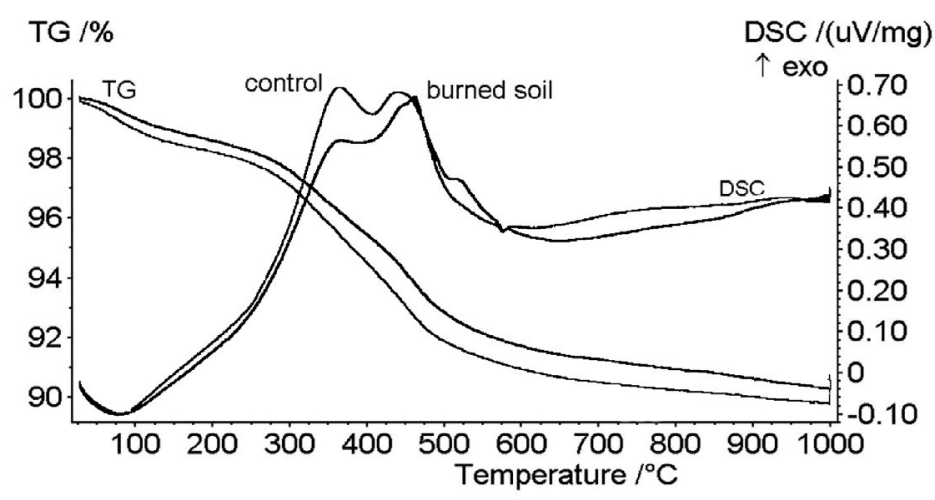

Tanto $C$ como $Q$ presentaban similares condiciones fisiográficas y ambientales

Figura 3. Termogramas de pérdida de peso (TGs) y calorimetría diferencial de barrido (DSC) de muestras de suelos afectados $(Q)$ y no afectados $(C)$ por un incendio en la Sierra de Alájar (Huelva,

España). Tanto $Q$ como $C$ presentaban similares condiciones fisiográficas y ambientales. 
En general, en suelos afectados por incendios se presenta un incremento en la MO de mayor recalcitrancia, a expensas normalmente de las fracciones más lábiles (MO libre y ácidos fúlvicos), efectos comparables a los procesos naturales de maduración, y que normalmente se reconocen por un aumento del grado de polimerización y de humificación. Sin embargo, estos cambios en la estructura química no pueden ser observados mediante técnicas de análisis térmico, por ello la combinación de éstas con técnicas espectroscópicas (RMN ó FT-IR por ejemplo) ha sido considerada especialmente conveniente (Lammers et al. 2009; Nocentini et al., 2010; Francioso et al., 2011).

Recientemente se han publicado diversas investigaciones sobre el impacto del fuego en la fracción lipídica del suelo como indicador de la intensidad y del efecto residual de los incendios (González-Pérez et al., 2004; Wiessenberg et al., 2009). Los lípidos del suelo se definen desde un punto de vista operativo como sustancias insolubles en agua pero susceptibles de ser extraídas con disolventes no polares. Constituyen, por tanto, un grupo heterogéneo de compuestos, algunos de ellos altamente sensibles a los cambios ambientales (Almendros et al., 1988). Por ejemplo, los patrones de distribución de compuestos alquílicos (fundamentalmente alcanos y ácidos grasos) se ven modificados por efecto del fuego. Concretamente, se observa un enriquecimiento relativo en homólogos de bajo peso molecular $\left(<\mathrm{C}_{20}\right)$, y una profunda alteración en el típico patrón consistente en el predominio de cadenas de número impar de átomos $\mathrm{C}$ (en el caso de alcanos) o de número par de C (en los ácidos grasos) (Fig. 4). Las relaciones entre la abundancia de determinados compuestos lipídicos se han mostrado como índices válidos para representar la recuperación ambiental de suelos afectados por incendios (González-Pérez et al., 2008).

En cuanto al contenido total de lípidos del suelo tras el efecto del incendio, los resultados dependerán en gran medida de las condiciones particulares del fuego, con mayor o menor aporte de biomasa parcialmente quemada. Se han descrito incrementos en la proporción de lípidos en los incendios de media y baja intensidad, debido a la incorporación al suelo de sustancias provenientes de la quema de hojarasca y biomasa vegetal, mientras que la concentración de lípidos puede verse reducida significativamente en incendios de alta intensidad donde la biomasa es completamente carbonizada.

En cualquier caso, es necesario resaltar que incluso en los casos en que el contenido de lípidos disminuye, la hidrofobicidad puede aumentar muy significativamente. Esto sugiere que la repelencia al agua depende en gran medida de los cambios en las propiedades coloidales y de superficie de la MO, a lo que se une la posible condensación o "fijación" de lípidos en estructuras macromoleculares no extraíbles (Almendros et al., 2010), y no necesariamente a la acumulación de sustancias lipídicas extraíbles.

En la actualidad se están desarrollando otras herramientas analíticas para avanzar en el estudio de los efectos del fuego en la MOS. Por ejemplo la respuesta de los valores de $\delta^{13} \mathrm{C}$ de la MOS afectada por el fuego está siendo objeto de debate (Saito et al., 2007; Huber et al., 2013). Algunos autores han observado un incremento relativo en los valores de $\delta^{13} \mathrm{C}$ en muestras de suelos quemados (Bowman et al., 2007; Alexis et al., 2010); sin embargo otras investigaciones muestran la falta de una tendencia clara (Czimczik et al., 2002). 

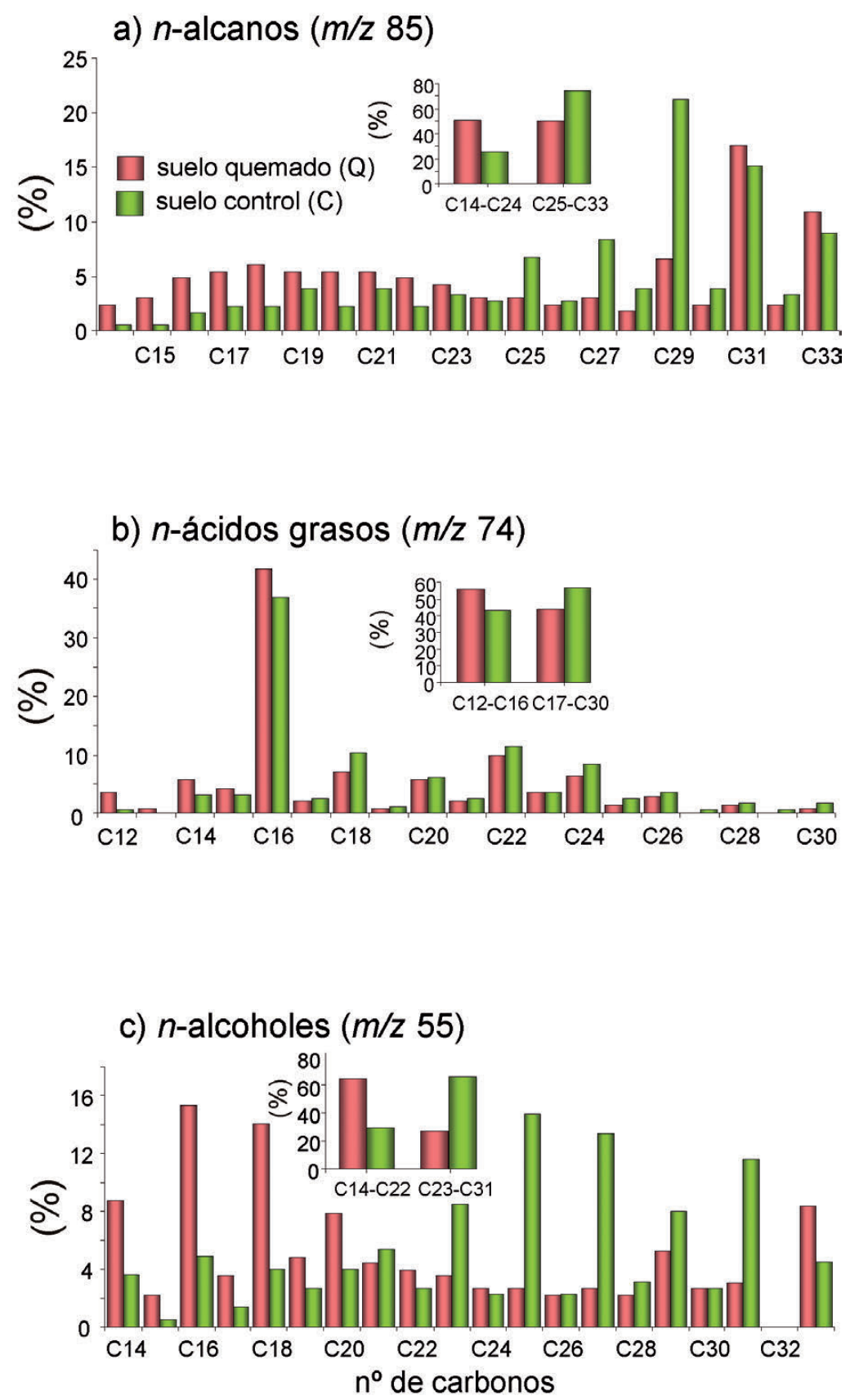

Figura 4. Distribución de compuestos alquílicos extraídos de la fracción lipídica de suelos Rendollic Xerochrept de la Sierra de Cazorla (Jaén, España). Tanto Q como C presentaban similares condiciones fisiográficas y ambientales. 
Temperatura ${ }^{\circ} \mathrm{C}$

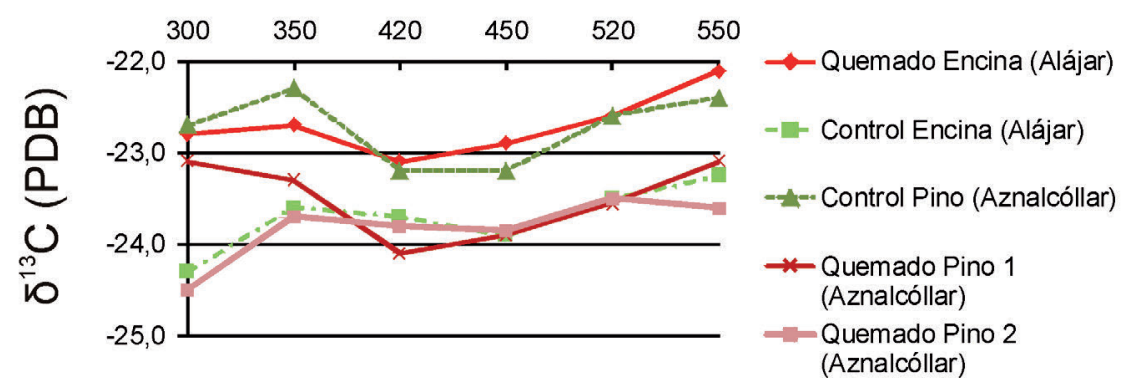

Figura 5. Valores de $\delta^{13} \mathrm{C}$ de muestras de suelos control y quemado medidos a 300, 350, $420,450,520$ y $550^{\circ} \mathrm{C}$ durante la degradación térmica realizada mediante termogravimetría acoplada a espectrometría de masas de isótopos estables (TG-IRMS).

La Fig. 5 muestra los cambios en la medida de $\delta^{13} \mathrm{C}$ de la MOS a distintas temperaturas en suelos quemados y control bajo pino y encina procedentes de bosques típicamente Mediterráneos del suroeste de la Península Ibérica. Independientemente de si el suelo había sido afectado o no por el fuego, se observa una disminución relativa en los valores de $\delta^{13} \mathrm{C}$ a temperaturas superiores de $420^{\circ} \mathrm{C}$, sin embargo a temperaturas más bajas el comportamiento de los valores de $\delta^{13} \mathrm{C}$ frente a los cambios de temperatura, vegetación o presencia del fuego no sigue un patrón reconocible. Estos resultados indican que probablemente hay múltiples procesos interrelacionados en cada una de las etapas de la alteración térmica de la MO. De hecho, es de sobra conocida la utilización de los valores de $\delta^{13} \mathrm{C}$ en estudios geocronológicos y arqueológicos, de tal forma que nuestros resultados sugieren que se debe tener en cuenta la incertidumbre que los cambios de temperatura producen en la interpretación de los resultados.

\section{Cambios en la biota del suelo}

El fuego ejerce también efectos significativos sobre la biota del suelo, que pueden afectar a la evolución de la MO. Estos cambios son muy complejos, y dependen de diversos factores que interactúan entre sí como son el grado de esterilización del suelo, la formación de cenizas, la formación de MO recalcitrante, el desarrollo de microflora secundaria y otros factores adicionales que regulan la evolución del suelo (Vega et al., 2013). A modo de resumen, se podría decir que el fuego suele causar a corto plazo una drástica reducción de la biomasa microbiana del suelo (Villar et al., 2004). Por lo general, las bacterias se muestran más resistentes que los hongos a las condiciones post-fuego, produciéndose frecuentemente un incremento en la población bacteriana (Guénon et al., 2013). Igualmente, tras un incendio, suele tener lugar un incremento en la concentración de nutrientes asimilables (mayoritariamente en forma de componentes de la cenizas solubles en agua), que estarán disponibles para los organismos (Bárcenas-Moreno et al., 2011). Este efecto "fertilizador" del fuego, que está ligado al incremento en el pH del suelo y al de cationes intercambiables es de sobra conocido desde los inicios de la agricultura. 
En cuanto a la estabilidad microbiológica del "black carbon”, se sabe que éste puede ser biodegradado en un tiempo razonable especialmente cuando se encuentra finamente dividido y en condiciones ambientales favorables para la biomasa microbiana (De la Rosa et al., 2011), sin embargo, el "black carbon" es considerado como altamente recalcitrante y desde el punto de vista experimental es muy difícil observar cualquier tipo de asimilación microbiana del mismo.

\section{Conclusiones}

Los efectos del fuego en la MOS dependen en gran medida de la severidad del incendio y de las condiciones ambientales. En general, los incendios reducen las reservas de MOS, por su efecto directo (volatilización) o por la interrupción temporal de los aportes. Por otra parte, las alteraciones en la composición molecular tras un incendio son importantes, entre las que cabe destacar:

i) Cambios en la composición molecular de las fracciones de lípidos libres del suelo, reducción de la longitud media de las cadenas de los compuestos alquílicos, tales como $n$-alcanos, alcoholes o ácidos grasos lineales, ii) cambios en las formas de MO de elevado peso molecular, con aromatización de polisacáridos y lípidos, iii) formación de compuestos heterocíclicos nitrogenados, lo que define de forma característica la composición de las sustancias húmicas presentes en suelos afectados por el fuego respecto a los no quemados, y iv) incremento en la fracción de MO más recalcitrante, asociada al enriquecimiento en formas de MO altamente condensadas (black carbon). En este sentido, los incendios forestales pueden tener una especial trascendencia en los mecanismos naturales de secuestro de C y N a largo plazo en los ecosistemas Mediterráneos. Sin embargo, estudios recientes cuestionan la estabilidad a largo plazo de la MO pirogénica en el suelo.

El uso combinado de herramientas espectroscópicas, cromatográficas y análisis térmico se ha mostrado como la forma más completa de discernir los cambios a nivel cualitativo de la MOS.

A pesar de los avances realizados en el estudio de los efectos del fuego en la MOS, existen todavía varios aspectos de los que se conoce muy poco y que están generando nuevas líneas de investigación: i) establecimiento de indicadores e índices que permitan predecir la recuperación post-incendio, que sean válidos para escenarios ambientales diversos, ii) diseño de metodologías apropiadas para evaluar la estabilidad y biodegradabilidad del "black carbon" formado, y iii) desarrollo de nuevas herramientas válidas para una rápida diagnosis de los cambios estructurales y funcionales en la MOS.

\section{Agradecimientos}

Nuestro agradecimiento a la Dra. Elisa López Capel (School of Civil Engineering and Geosciences, Newcastle University) por su colaboración en el análisis térmico (TG y DSC) de los suelos. El Dr. J.M. de la Rosa agradece al Ministerio de Economía y Competitividad y al Consejo Superior de Investigaciones Científicas la financiación de su contrato JAE-DOC. 


\section{Referencias bibliográficas}

Alexis, M.A., Rumpel, C., Knicker, H., Leifeld, J., Rasse, D., Péchot, N., Bardoux, G., Mariotti, A. 2010. Thermal alteration of organic matter during a shrubland fire: a field study. Organic Geochemistry 41, 690-697.

Almendros, G., Gonzalez-Vila, F.J. 2012. Wildfires, soil carbon balance and resilient organic matter in Mediterranean ecosystems. A review. Spanish Journal of Soil Science 2 (2), 8-33.

Almendros, G., Polo, A., Lobo, M.C., Ibáñez, J. 1984. Contribución al estudio de la influencia de los incendios forestales en las características de la materia orgánica del suelo. II: Transformaciones del humus por ignición en condiciones controladas de laboratorio. Revue d'Ecologie et de Biologie du Sol 21, 145-160.

Almendros, G., Martín, F., González-Vila, F.J. 1988. Effects of fire on humic and lipid fractions in a Dystric Xerochrept in Spain. Geoderma 42, 115-127.

Almendros, G., González-Vila, F.J., Martin, F., Fründ, R., Lüdemann H.D. 1992. Solid state NMR studies of fire-induced changes in the structure of humic substances. Science of the Total Environment 117-118, 63-74.

Almendros, G., Knicker, H., González-Vila, F.J. 2003. Rearrangement of carbon and nitrogen forms in peat after progressive isothermal heating as determined by solid-state ${ }^{13} \mathrm{C}$ - and ${ }^{15}$ N-NMR spectroscopies. Organic Geochemistry 34, 1559-1568.

Almendros, G., González-Vila, F.J., Knicker, H., Verdejo, T., González-Pérez, J.A., Dettweiler, J.A. 2010. Reappraisal of water-repellence-inducing soil organic material in mineral soils after controlled thermal heating. En Investigación y gestión para la protección del suelo y restauración de los ecosistemas afectados por incendios forestales, Fuegored 2010, Santiago de Compostela, pp. 91-94.

Arias, M.E., González-Pérez, J.A., González-Vila, F.J., Ball, A.S. 2005. Soil health-a new challenge for microbiologists and chemists. International Microbiology 8, 13-21.

Bárcenas-Moreno, G., García-Orenes, F., Mataix-Solera, J., Mataix-Beneyto, J., Baath, E. 2011. Soil microbial recolonisation after a fire in a Mediterranean forest. Biology and Fertility of Soils 47, 261-272.

Barros, N., Salgado, J., Villanueva, M., Rodriquez-Anon, J., Proupin, J., Feijoo, S., Martin-Pastor, M. 2011. Application of DSC-TG and NMR to study the soil organic matter. Journal of Thermal Analysis and Calorimetry 104, 53-60.

Bodí, M.B., Cerdà, A., Mataix-Solera, J., Doerr, S.H. 2012. Repelencia al agua en suelos forestales afectados por incendios y en suelos agrícolas bajo distintos manejos y abandono. Cuadernos de Investigacion Geográfica 38 (2), 53-74.

Bowman, D.M., Boggs, G.S., Prior, L.D., Krull, E.S. 2007. Dynamics of Acacia aneura-Triodia boundaries using carbon (C-14 and delta C-13) and nitrogen (delta $\mathrm{N}-15)$ signatures in soil organic matter in central Australia. The Holocene 17, 311-318.

Certini, G. 2005. Effects of fire on properties of forest soils: a review. Oecologia 143, 1-10.

Chandler, C., Cheney, P., Thomas, P., Trabaud, L., Williams, D. 1983. Forest Fire Behavior and Effects. Fire in Forestry, vol I. Wiley, New York, pp 171-202.

Czimczik, C.I., Preston, C.M., Schmidt, M.W.I., Werner, R.A., Schulze, E.D. 2002. Effects of charring on mass, organic carbon, and stable carbon isotope composition of wood. OrganicGeochemistry 33, 1207-1223.

De la Rosa, J.M., Knicker, H. 2011. Bioavailability of N released from pyrogenic organic matter: An incubation study. Soil Biology and Biochemistry 43, 2368-2373.

De la Rosa, J.M., González-Pérez, J.A., González-Vázquez, R., Knicker, H., López-Capel, E., Manning, D.A.C., González-Vila, F.J. 2008a. Use of pyrolysis/GC-MS combined with 
thermal analysis to monitor $\mathrm{C}$ and $\mathrm{N}$ changes in soil organic matter from a Mediterranean fire affected forest. Catena 74, 296-303.

De la Rosa, J.M., Lopez Capel, E., González-Vila, F.J., González-Perez, J.A., Manning, D.A.C. 2008b. Direct detection of black carbon in soils by Py-GC/MS, ${ }^{13} \mathrm{C}$ NMR spectroscopy and thermogravimetric techniques. American Journal of Soil Science Society 72, 258-267.

De la Rosa, J.M., González-Pérez, J.A., Knicker, H., López-Capel, E., Manning, D.A.C., GonzálezVila, F.J. 2009. Structural properties of non combustion derived refractory organic matter which interfere with BC quantification. Journal of Analytical and Applied Pyrolysis 85, 299-308.

De la Rosa, J.M., Varela, M.E., Faria, S.R., González-Vila, F.J., Knicker, H., González-Pérez, J.A., Keizer, J., 2012. Characterization of wildfire effects on soil organic matter using analytical pyrolysis. Geoderma 191, 24-30.

Doerr, S.H., Woods, S.W., Martin, D.A., Casimiro, M. 2009. Natural background' soil water repellency in conifer forests of the north-western USA: Its prediction and relationship to wildfire occurrence. Journal of Hydrology 37, 12-21.

Fernández, J.M., Plante, A.F., Leifeld, J., Rasmussen, C. 2011. Methodological considerations for using thermal analysis in the characterization of soil organic matter. Journal of Thermal Analysis and Calorimetry 104, 389-398.

Francioso, O., Sánchez-Cortés, S., Bonora, S., Roldán, M.L., Certini, G. 2011. Structural characterization of charcoal size-fractions from a burnt Pinus pinea forest by FT-IR, Raman and surface-enhanced Raman spectroscopies. Journal of Molecular Structure 994, 155-162.

González-Pérez, J.A., González-Vila, F.J., Almendros, G., Knicker, H. 2004.The effect of fire on soil organic matter-a review. Environment International 30, 855-870.

González-Pérez, J.A., González-Vila, F.J., González-Vázquez, R., Arias, M.E., Rodríguez, J., Knicker, H. 2008. Use of multiple biogeochemical parameters to monitor the recovery of soils after forest fires. Organic Geochemistry 39, 940-944.

González-Vila, F.J., Almendros, G. 2013. El controvertido efecto de los incendios: El fuego en el ciclo del carbono en los ecosistemas mediterráneos. En Cuando se quema el bosque. El impacto del fuego en los ecosistemas, Mètode, pp. 83-87.

Guénon, R., Vennetier, M., Dupuy, N., Roussos, S., Pailler, A., Gros, R. 2013.Trends in recovery of Mediterranean soil chemical properties and microbial activities after infrequent and frequent wildfires. Land Degradation and Development 24, 115-128.

Hilscher, A., Knicker, H. 2011.Carbon and nitrogen degradation on molecular scale of grassderived pyrogenic organic material during 28 months of incubation in soil. Soil Biology and Biochemistry 43, 261-270.

Huber, E., Bell, T.L., Adams, M.A. 2013. Combustion influences on natural abundance nitrogen isotope ratio in soil and plants following a wildfire in a sub-alpine ecosystem. Oecologia 173, 1063-1074.

Johnson, D.W., Curtis, P.S. 2001. Effects of forest management on soil C and N storage: metaanalysis. Forest Ecology and Management 140, 227-238.

Kavdır, Y., Ekinci, H., Yüksel, O., Mermut, A.R. 2005. Soil aggregate stability and ${ }^{13} \mathrm{C}$ CP/MASNMR assessment of organic matter in soils influenced by forest wildfires in Çanakkale, Turkey. Geoderma 129, 219-229.

Knicker, H. 2000. Biogenic nitrogen in soils as revealed by solid-state carbon-13 and nitrogen-15 nuclear magnetic resonance spectroscopy. Journal of Environmental Quality 29, 715-723.

Knicker, H. 2007. How does fire affect the nature and stability of soil organic nitrogen and carbon? A review. Biogeochemistry 85, 91-118.

Knicker, H., González-Vila, F.J., Polvillo, O., González-Pérez, J.A., Almendros, G. 2005. Fire-induced transformation of $\mathrm{C}$ - and $\mathrm{N}$ - forms in different organic soil fractions from 
a DystricCambisol under a Mediterranean pine forest (Pinus pinaster). Soil Biology \& Biochemistry 37, 701-707.

Kuhlbusch, T.A.J.,Crutzen, P.J. 1995. Toward a global estimate of black carbon in residues of vegetation fires representing a sink of atmospheric $\mathrm{CO}_{2}$ and a source of $\mathrm{O}_{2}$. Global Biogeochemical Cycles 9, 491-501.

Lammers, K., Arbuckle-Keil, G., Dighton, J. 2009. FT-IR study of the changes in carbohydrate chemistry of three New Jersey pine barrens leaf litters during simulated control burning. Soil Biology \& Biochemistry 41, 340-347.

Lasheras-Álvarez, L., Pérez-Sanz, A., Gil-Romera, G., González-Sampériz, P., Sevilla-Callejo, M., Valero-Garcés, B.L. 2013. History of fire and vegetation in a Holocene sequence of the Central Pyrenees: the Basa de la Mora Lake. Cuadernos de Investigación Geográfica 39, 77-95.

Leifeld, J. 2007. Thermal stability of black carbon characterised by oxidative differential scanning calorimetry. Organic Geochemistry 38, 112-127.

Lützow M.V., Kögel-Knabner, I. Ekschmitt, K. Matzner, E. Guggenberger, G. Marschner, B. Flessa, H. 2006. Stabilization of organic matter in temperate soils: mechanisms and their relevance under different soil conditions - a review. European Journal of Soil Science 57, 426-444.

Martín, A., Díaz-Raviña, M., Carballas, T. 2012. Short- and medium-term evolution of soil properties in Atlantic forest ecosystems affected by wildfires. Land Degradation \& Development $23,427-439$.

Moriondo, M., Good, P., Durao, R., Bindi, M., Giannakopoulos, C., Corte-Real, C. 2006. Potential impact of climate change on fire risk in the Mediterranean area. Climate Research 31, 85-95.

Nocentini, C., Certini, G., Knicker, H., Francioso, O., Rumpel, C. 2010. Nature and reactivity of charcoal produced and added to soil during wildfire are particle-size dependent. Organic Geochemistry 41, 682-689.

Parker, J.L., Fernandez, I.J., Rustad, L.E., Norton, S.A. 2001.Effects of nitrogen enrichment, wildfire, and harvesting on forest-soil carbon and nitrogen. Soil Science Society of America Journal 65, 1248-1255.

Pausas, J.G., Llovet, J., Rodrigo, A., Vallejo, R. 2008. Are wildfires a disaster in the Mediterranean basin?-A review. International Journal of Wildland Fire 17, 713-723.

Plante, A.F., Fernández, J.M., Leifeld, J. 2009. Application of thermal analysis techniques in soil science. Geoderma 153, 1-10.

Reeves III, J.B., McCarty, G.W., Rutherford, D.W., Wershaw, R.L. 2008. Mid-infrared diffuse reflectance spectroscopic examination of charred pine wood, bark, cellulose, and lignin: Implications for the quantitative determination of charcoal in soils. Applied Spectroscopy 62, 182-189.

Rovira, P., Romanyà, J., Duguy B. 2012. Long-term effects of wildfires on the biochemical quality of soil organic matter: A study on Mediterranean shrublands. Geoderma 179-180, 9-19.

Rumpel, C., González-Pérez, J.A., Bardoux, G., Largeau, C., Gonzalez-Vila, F.J., Valentín, C. 2007. Composition and reactivity of morphologically distinct charred materials left after slash-and-burn practices in agricultural tropical soils. Organic Geochemistry 38, 911-920.

Saito, L., Miller, W.W., Johnson, D.W., Qualls, R.G., Provencher, L., Carroll, E., Szameitat, P. 2007. Fire effects on stable isotopes in a Sierran forested watershed. Journal of Environmental Quality 36, 91-100.

Stevenson, F.J. 1994. Humus Chemistry: Genesis, Composition, Reactions. 2nd ed., Wiley, New York. 
Tinoco, P., Almendros, G., Sanz, J., González-Vázquez, R., González-Vila, F.J. 2006. Molecular descriptors of the effect of fire on soils under pine forest in two continental Mediterranean soils. Organic Geochemistry 37 (12), 1995-2018.

Vega, J.A., Fontúrbel, T., Merino, A., Fernández, C., Ferreiro, A., Jiménez, E. 2013. Testing the ability of visual indicators of soil burn severity to reflect changes in soil chemical and microbial properties in pine forests and shrubland. Plant and Soil 369, 73-91.

Villar, M.C., Petrikova, V., Díaz-Raviña, M., Carballas, T. 2004. Changes in soil microbial biomass and aggregate stability following burning and soil rehabilitation. Geoderma 122, 73825

Wander, M. 2004. Soil organic matter fractions and their relevance to soil function. En Soil organic matter in sustainable agriculture, RM. Magdoff, R.R. Weil (eds.), CRC Press, Boca Raton, Florida.

Wiesenberg, G.L.B., Lehndorff, E., Schwark, L. 2009. Thermal degradation of rye and maize straw: Lipid pattern changes as a function of temperature. Organic Geochemistry 40, 167174. 